\title{
Hydrophilic Microporous Layer Coatings for Polymer Electrolyte Membrane Fuel Cells
}

\author{
Pranay Shrestha, Rupak Banerjee, Jongmin Lee, Aimy Bazylak \\ Thermofluids for Energy and Advanced Materials (TEAM) Laboratory \\ Department of Mechanical \& Industrial Engineering, University of Toronto Institute for Sustainable Energy \\ Faculty of Applied Science \& Engineering, University of Toronto \\ 5 King's College Road, Toronto, Ontario M5S 3G8, Canada \\ abazylak@mie.utoronto.ca
}

\begin{abstract}
Polymer electrolyte membrane (PEM) fuel cells are energy conversion devices that are promising components of a sustainable energy system. However, conventional fuel cells suffer from performance degradation at low humidification. To address poor performance under low humidity conditions, we investigate the effect of custom hydrophilic microporous layer (MPL) coatings on liquid water distributions in gas diffusion layers (GDLs), observed in operando using X-ray synchrotron radiography. The visualization was performed during low humidity operating conditions at $60^{\circ} \mathrm{C}$, with inlet gas relative humidity (RH) of 50\%. Hydrophilic MPLs were coated onto commercial hydrophobic GDLs, and electrical output and simultaneous liquid water measurements were measured, using a fuel cell test station and X-ray synchrotron radiography respectively. The hydrophilic materials showed lower values of membrane resistance (quantified by high frequency resistance) compared to the benchmark hydrophobic GDL. The relatively lower membrane resistance was attributed to improved membrane hydration under low humidity conditions. Correspondingly, the catalyst layer (CL)-MPL interface contained larger quantities of liquid water with the addition of the hydrophilic coating. However, excess liquid water at the cathode side of the GDL led to high oxygen mass transport losses at high current densities. Understanding gained from this study can be used to optimize wettability of the MPL to operate the fuel cell at low or no external humidification.
\end{abstract}

Keywords: PEM Fuel Cells, Hydrophilic Microporous Layer, Liquid Water Visualization, Low Humidity

\section{Introduction}

Polymer electrolyte membrane (PEM) fuel cells serve as promising energy conversion devices that are particularly attractive for sustainable automotive applications [1]. Water management in the PEM fuel cell is critical for balancing two competing needs: a) adequate hydration of the polymer electrolyte membrane for protonic conductivity, and b) preservation of oxygen mass transport pathways for fuel delivery by reducing excess water accumulation at the cathode GDL [2]. To hydrate the membrane and prevent "membrane dry-out", external humidifiers are conventionally incorporated into the fuel cell system. However, humidifiers add to the system cost, weight, and parasitic power demands [3], and removing or reducing humidification would greatly simplify the fuel cell system [4]. To prevent the accumulation of excess liquid water, a hydrophobic microporous layer (MPL) is included between the gas diffusion layer (GDL) and catalyst layer of a PEM fuel cell [5]-[7]. However, the performance of conventional fuel cells typically suffers in the absence or reduction of humidification of inlet gases [8].

Several recent studies have shown that hydrophilic MPLs provide performance improvements compared to conventional hydrophobic materials under low humidity operating conditions [9]-[16]. Kitahara et al. [9]-[12] investigated a multi-layered MPL with controlled hydrophilicity, and they showed that coating a $5 \mu \mathrm{m}$ hydrophilic MPL between a hydrophobic MPL and the catalyst layer resulted in improved fuel cell performance at low humidity conditions. Tanuma et al. [13], [14] demonstrated that a hydrophilic MPL, consisting of vapor-grown carbon fibers and ionomer, facilitated favourable fuel cell performance at both dry and wet conditions. Ahn et al. [15] showed that a hydrophilic MPL with carbon black and Nafion ${ }^{\circledR}$ ionomer led to improved fuel cell performance when placed at the cathode. The dominating mechanisms by which hydrophilic MPLs influence water distribution in the GDL still needs to be explained.

$\mathrm{X}$-ray synchrotron radiography is a powerful tool for visualizing liquid water in the opaque fuel cell during operation, with high spatial and temporal resolution [17]-[19] Some authors have visualized liquid water in the GDL containing 
hydrophilic components using methods such as neutron imaging [20] and cryo-SEM [16]. Mukundan et al. [20] used neutron radiography to visualize liquid water in the GDL and showed that hydrophilic alumosilicate fibers in the MPL created wicking pathways for liquid water. Aoyama et al. [16] froze their GDL soon after discontinuing operation of the fuel cell, and they visualized the resulting ice using cryo-SEM. However, in operando visualization would provide new, clarifying insight into the effect of MPL hydrophilicity on liquid water transport.

In this study, we investigate the effect of custom hydrophilic MPL coatings on liquid water distributions in GDLs using in operando X-ray synchrotron radiography. The visualization was performed under low humidity conditions with inlet gas relative humidity $(\mathrm{RH})$ of $50 \%$ at $60^{\circ} \mathrm{C}$.

\section{Methodology}

\subsection{MPL Fabrication}

A commercial hydrophobic GDL (SGL 25BC, Sigracet@ Group) was modified in-house through the application of hydrophilic coatings. A hydrophilic layer was added near the CL side to improve membrane hydration under lowhumidity/ low current density operating conditions. The hydrophilic MPL slurry was composed of carbon black (Vulcan ${ }^{\circledR}$ XC-72R, Fuel Cell Store), deionized water, surfactant (Triton ${ }^{\mathrm{TM}}$ X-100, Sigma-Aldrich), and varying levels of dispersion of perfluorosulphonic acid ionomer (Nafion ${ }^{\circledR}$ dispersion D1021, IonPower) in the ratio of 1: 10: 0.2: 0.01 (solid) and 1: 10: 0.2: 0.02 (solid) by weight for two GDLs. The slurry was mixed using a magnetic stirrer and sonicated for 60 minutes at 50\% amplitude using a Q125 sonicator with a CL-18 tip (Q Sonica, LLC.). The slurry was coated with a $5 \mu \mathrm{m}$ thickness onto the SGL 25BC (on the MPL side) using the doctor blade method [21]. The custom hydrophilic layer was dried at an RH of $98 \%$ in an environmental chamber (Tenney C-EVO Temperature / Humidity Test Chamber) for 3.5 days in an effort to reduce the speed of the drying process. The MPL was then dried at $250^{\circ} \mathrm{C}$ for 1 hour and sintered at $350^{\circ} \mathrm{C}$ for 1 hour in a convection oven (DHG9000JB, MTI Corporation). The details of the GDL materials tested are shown in Table 1.

Table 1: Details of the three GDL materials used in MEAs that were tested in the fuel cell. Custom hydrophilic coating was applied to two GDLs to vary hydrophilicity of MPL at catalyst layer-MPL interface.

\begin{tabular}{|l|l|l|}
\hline \multicolumn{1}{|c|}{ GDL name } & Anode and Cathode GDL & Custom MPL coating \\
\hline Hydrophobic MPL & SGL 25 BC & None \\
\hline Hydrophilic MPL coating 1 & SGL 25 BC (base) & $\begin{array}{l}\text { Hydrophilic MPL with Nafion® } \\
\text { 1wt.\% of carbon black }\end{array}$ \\
\hline Hydrophilic MPL coating 2 & SGL 25 BC (base) & $\begin{array}{l}\text { Hydrophilic MPL with Nafion® } \\
\text { 2wt.\% of carbon black }\end{array}$ \\
\hline
\end{tabular}

\subsection{Fuel Cell Testing}

A custom fuel cell with parallel flow field channels (of $0.5 \mathrm{~mm}$ width and depth) and an active area of $0.68 \mathrm{~cm}^{2}$ $(0.85 \mathrm{~cm} \times 0.80 \mathrm{~cm})$ was used to simultaneously monitor the performance and water thickness of the accumulated liquid water. The membrane electrode assembly (MEA) consisted of a catalyst coated membrane (CCM), i.e. Nafion ${ }^{\circledR}$ membrane HP (Ion Power) coated with Pt/C catalyst layers (with platinum loading of $0.30 \mathrm{mg} / \mathrm{cm}^{2}$ ), sandwiched by anode and cathode GDLs that were compressed to 78-80\% of their original thickness using polyethylene naphthalate (PEN) spacers. The fuel cells were controlled using a fuel cell test station (Scribner 850e, Scribner Associates Inc.). The fuel cell temperature was maintained at $60^{\circ} \mathrm{C}$ with water cooled end plates. Hydrogen and air were supplied at 1 slpm with a back pressure of $100 \mathrm{kPa}$ (gauge). The gas inlet relative humidity was set to $50 \%$ (with a dew point temperature of $46^{\circ} \mathrm{C}$ ). Constant current steps of $0.25,0.50,1.00,1.50,2.00,2.50 \mathrm{~A} / \mathrm{cm}^{2}$ were held for 15 minutes each.

\subsection{X-ray Synchrotron Visualization}

The Biomedical Imaging and Therapy Bending Magnet (05B1-1) beamline facility at the Canadian Light Source (CLS) in Saskatoon, Canada [22] was used to visualize liquid water during fuel cell operation. A monochromatic collimated X-ray beam of $24 \mathrm{keV}$ was used, and an AA-40 scintillator was combined with a Hamamatsu ORCA Flash 4.0 to obtain a pixel resolution of $6.5 \mu \mathrm{m} / \mathrm{pixel}$ and temporal resolution of $3 \mathrm{~s} /$ frame. 
The obtained images were corrected for beam intensity change, cell movement, and background camera noise. The test images were normalized with a reference averaged "dry" image from open circuit conditions. The Beer-Lambert law was used to interpret image intensity as the content of liquid water (liquid water thickness in the direction of the beam). We normalized this liquid water thickness by the extent of the GDL traversed by the beam (8mm). To capture the inhomogeneity of water profiles under the ribs and the channels of the flow-field, normalized water thickness values under the channels and the ribs were averaged and presented separately. For more details regarding the image processing procedure, the reader is referred to Ge et al. [18] and Hinebaugh et al. [23].

\section{Results and Discussion}

\subsection{Effect of Hydrophilic Coating on Membrane Resistance}

Fuel cell electrical output data for the three GDLs at 50\% inlet RH is shown in Figure 1. The voltage vs. current density curves show that the MEA with the hydrophobic MPL had the highest overpotential among the GDLs at the constant current steps. Correspondingly, the hydrophobic MPL had the highest membrane resistance indicated by high frequency resistance (HFR) values. Higher membrane resistance indicated that the membrane was less hydrated for the hydrophobic case under low humidity conditions. The addition of the hydrophilic layer led to enhanced membrane hydration and fuel cell performance (see also power density curves) under low humidity conditions. However, the GDL with hydrophilic coating 2 exhibited higher mass transport resistance at $2 \mathrm{~A} / \mathrm{cm}^{2}$ and reached a limiting current before reaching the $2.5 \mathrm{~A} / \mathrm{cm}^{2}$ current step.

a)

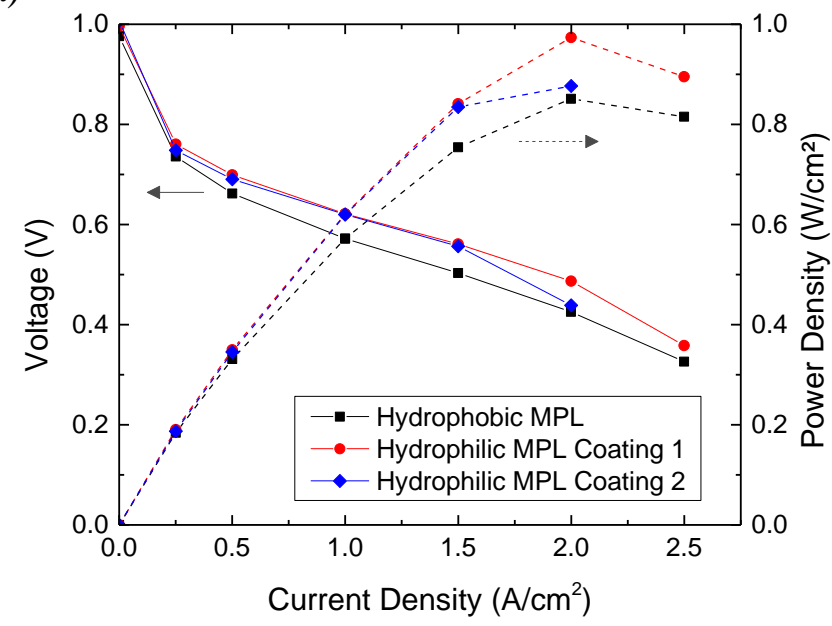

b)

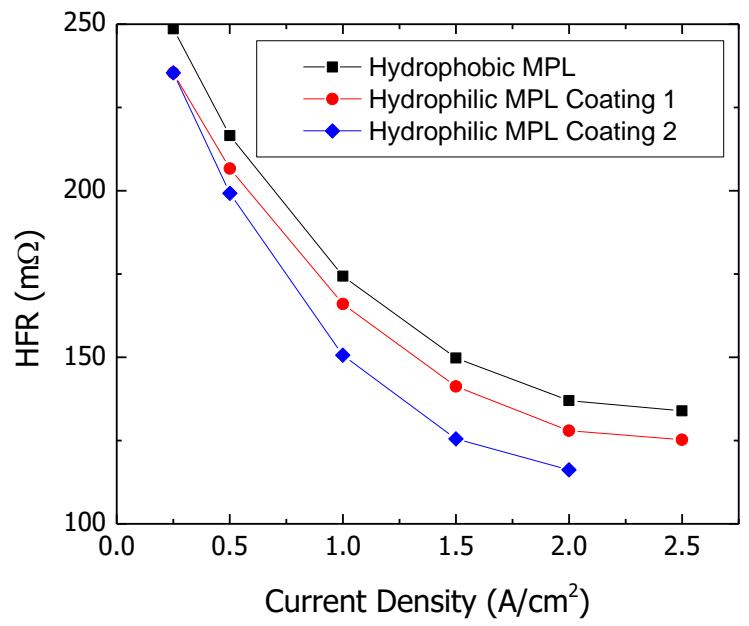

Fig. 1: Performance curves for MEAs with hydrophobic MPL, and hydrophilic MPL coatings 1 and 2 obtained at anode and cathode inlet RH of $50 \%$. a) Voltage vs. current density graphs showed higher overpotential for the hydrophobic case compared to the two hydrophilic cases. Power density vs. current density curves showed that hydrophilic coatings helped improve maximum power density under low humidity conditions b) High frequency resistance (HFR) vs. current density graph showed high values of high frequency resistance for the hydrophobic material that indicated low membrane hydration.

\subsection{Effect of Hydrophilic Coating on Liquid Water Accumulation in the GDL}

The normalized steady-state liquid water thickness profiles in the through plane direction are shown in Figures 2 and 3. At each current density, the cell was operated for 15 minutes to reach steady state and the water data was averaged for the last 5 minutes. Profiles for regions under the channels and the ribs of the flow-field are averaged and presented separately. The error bars represent the measurement uncertainty with a coverage factor of 3 , which corresponded to a confidence interval greater than $99 \%$. The reader is referred to [17] for more details on the procedure to calculate measurement uncertainty. The catalyst coated membrane is omitted from this study since we are focusing on the liquid water accumulation in the GDL materials. 

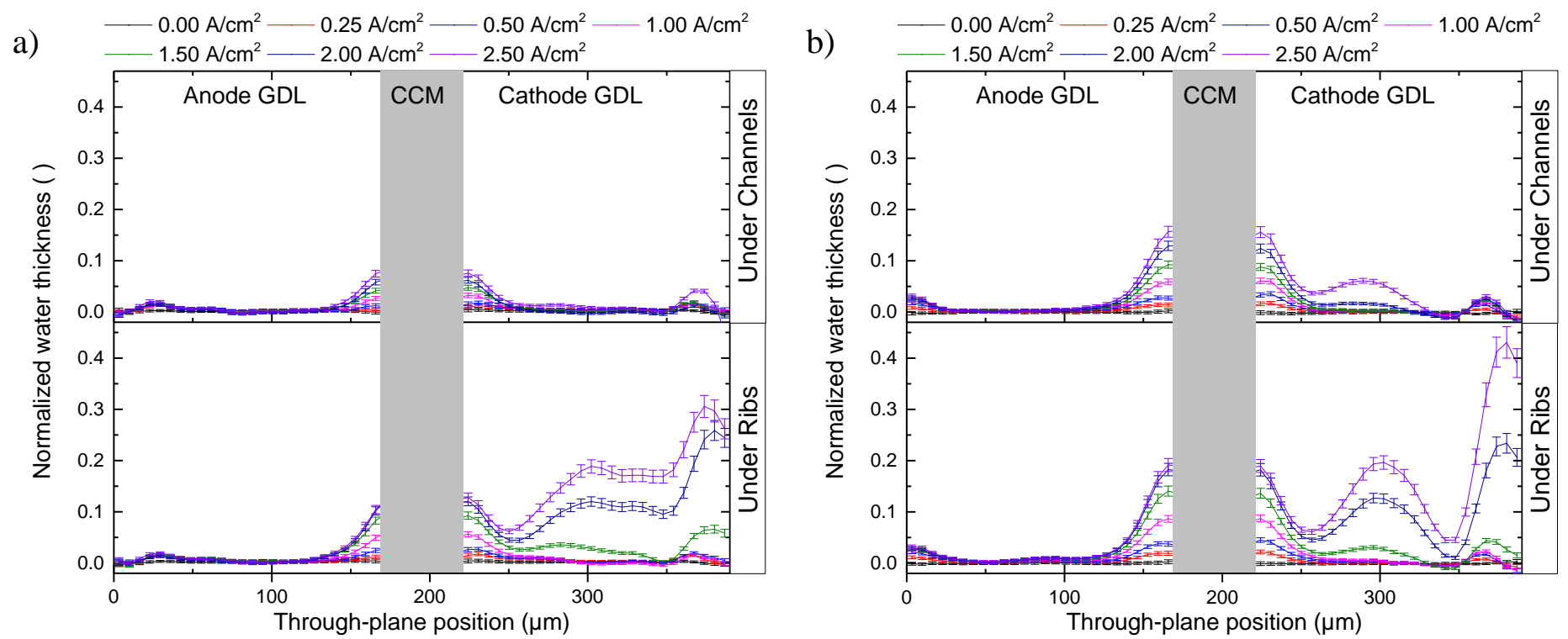

Fig. 2: Normalized water thickness vs. through-plane position for MEAs with a) hydrophobic MPL and b) hydrophilic MPL coating 1 obtained at anode and cathode inlet RH of 50\%. Regions under the flow-field channels and ribs are presented separately. The error bars represent the uncertainty of measurement with a coverage factor of 3 (>99\% confidence interval). The GDL with the hydrophilic coating showed higher water presence near the catalyst layer-MPL interface, that lead to better membrane hydration (as indicated by the high frequency resistance data). Liquid water content of the catalyst coated membrane (CCM) is omitted here.

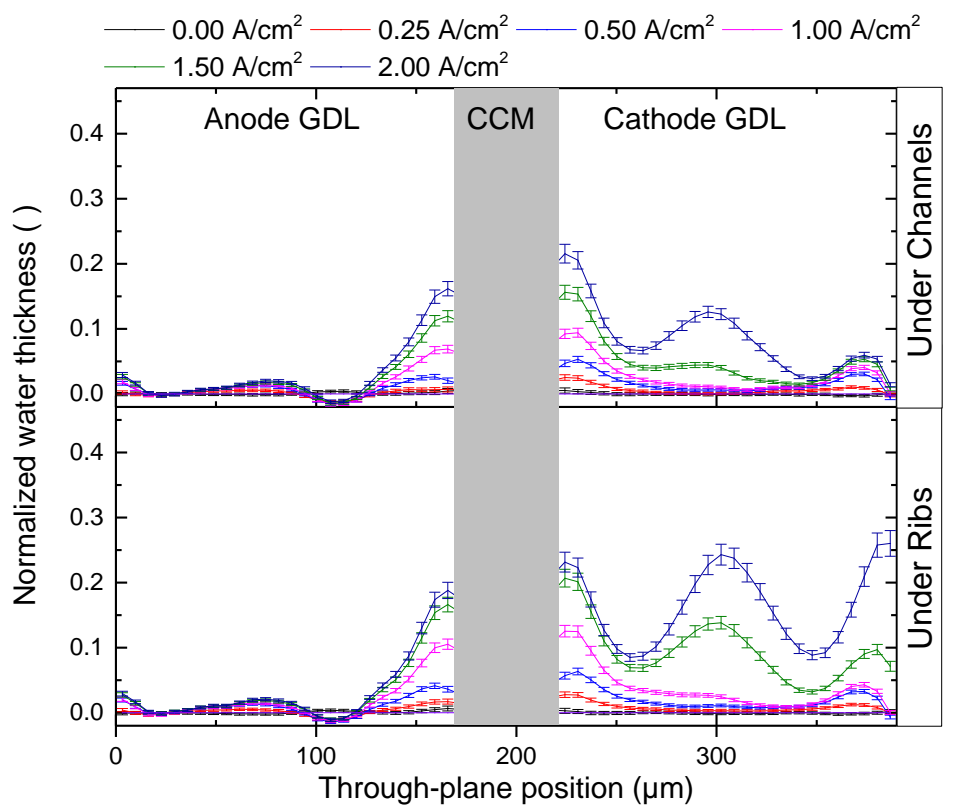

Fig. 3: Normalized water thickness vs. through-plane position for MEAs with hydrophilic MPL coating 2 obtained at anode and cathode inlet RH of 50\%. Regions under the flow-field channels and ribs are presented separately. The error bars represent the uncertainty of measurement with a coverage factor of 3 (>99\% confidence interval). Cathode GDL of MEA with hydrophilic MPL coating 2 showed higher quantity of liquid water at the catalyst layer-MPL interface and bulk substrate compared to the other materials at respective current density steps. The high quantity of liquid water corresponds to the high mass transport overpotential observed in performance curves.

Figure 2 shows that the hydrophilic layer was effective in retaining liquid water at the cathode catalyst layer-MPL interface. Nafion ${ }^{\circledR}$ has a low (hydrophilic) receding contact angle and high adhesion to water [24], so liquid water is 
expected to accumulate in the hydrophilic MPL (more than in the hydrophobic MPL). This accumulation of liquid water near the membrane promoted membrane hydration.

Figure 3 shows the normalized liquid water thickness profile for the GDL with hydrophilic coating 2. Higher water content was observed at the catalyst layer-MPL interface and the bulk substrate region for this GDL in comparison to the other GDLs, even at lower current densities (that corresponded to lower water production). High water accumulation (or flooding) at the cathode GDL was responsible for the high mass transport resistance at high current density (as seen in Figure 1(a)), since the pores that were saturated with water were unavailable for the transport of oxygen. Since MPL coating 2 had a higher Nafion ${ }^{\circledR}$ content than coating 1, coating 2 would have a stronger adhesion force to the water that was generated, which corresponds to higher water retention in the MPL.

\section{Conclusion}

In this study, we identified the mechanism by which hydrophilic MPL coatings enhance fuel cell performance at low humidification using synchrotron X-ray radiographic visualization of liquid water. Hydrophilic MPL coatings were shown to provide performance benefits over hydrophobic counterparts under low humidity condition (50\% inlet RH). Hydrophilic coatings facilitated the retention of more water at the cathode catalyst layer-MPL interface due to the high adhesion to product liquid water. Higher liquid water content at the catalyst layer-MPL interface was correlated with lower high frequency resistance measurements that indicated improved membrane hydration. Additionally, excess liquid water became more prevalent in the catalyst layer-MPL interface and bulk substrate when higher hydrophilic content was incorporated in the MPL coating. Excess liquid water accumulation in the cathode GDL led to high mass transport overpotential (losses) in the fuel cell, due to the blockage of pores available for oxygen diffusion. Understanding gained from this study can be applied towards designing GDLs better suited for low humidity operation of PEM fuel cells so that fuel cell systems with low or no external humidification can be operated at high power densities.

\section{Acknowledgements}

Financial support from the Natural Sciences and Engineering Research Council of Canada (NSERC), the NSERC Discovery Accelerator Program, the NSERC Canada Research Chairs Program, the Ontario Ministry of Research and Innovation Early Researcher Award, and the Canada Foundation for Innovation are gratefully acknowledged. Graduate scholarships to Pranay Shrestha from the Pierre Rivard Hydrogenics Graduate Fellowship are gratefully acknowledged. Graduate scholarships to Jongmin Lee from the Mercedes-Benz Canada Graduate Fellowship in Fuel Cell Research, the HATCH Graduate Scholarship, and the David Sanborn Scott \& Ron D. Venter Fellowship are gratefully acknowledged.

Research described in this paper was performed at the BMIT facility at the Canadian Light Source, which is supported by the Canada Foundation for Innovation, Natural Sciences and Engineering Research Council of Canada, the University of Saskatchewan, the Government of Saskatchewan, Western Economic Diversification Canada, the National Research Council Canada, and the Canadian Institutes of Health Research. Authors acknowledge the receipt of support from the CLS Post-Doctoral and Graduate Student Travel Support Program. The authors would like to acknowledge Dr. George Belev, Dr. Adam Webb, Dr. Ning Zhu, Dr. Tomasz Wysokinski, Denise Miller and the BMIT group of the Canadian Light Source Inc. for their generous assistance.

\section{References}

[1] Y. Wang et al, "A review of polymer electrolyte membrane fuel cells: Technology, applications, and needs on fundamental research," Appl. Energy, vol. 88, pp. 981-1007, 4, 2011.

[2] R. Banerjee et al, "Experimental validation of two-phase pressure drop multiplier as a diagnostic tool for characterizing PEM fuel cell performance," Int. J. of Hydrogen Energy, vol. 39, pp. 17791, 2014.

[3] F. N. Büchi and S. Srinivasan, "Operating proton exchange membrane fuel cells without external humidification of the reactant gases fundamental aspects," J. Electrochem. Soc., vol. 144, pp. 2767-2772, 1997.

[4] M. V. Williams, H. R. Kunz and J. M. Fenton, "Operation of Nafion®-based PEM fuel cells with no external humidification: influence of operating conditions and gas diffusion layers," J. Power Sources, vol. 135, pp. 122-134, 9/3, 2004.

[5] Z. Qi and A. Kaufman, "Improvement of water management by a microporous sublayer for PEM fuel cells," $J$. Power Sources, vol. 109, pp. 38-46, 2002. 
[6] P. Deevanhxay et al, "Effect of liquid water distribution in gas diffusion media with and without microporous layer on PEM fuel cell performance," Electrochemistry Communications, vol. 34, pp. 239-241, 2013.

[7] J. T. Gostick et al, "On the role of the microporous layer in PEMFC operation," Electrochemistry Communications, vol. 11, pp. 576-579, 2009.

[8] J. Yu et al, "Lifetime behavior of a PEM fuel cell with low humidification of feed stream," Physical Chemistry Chemical Physics, vol. 7, pp. 373-378, 2005.

[9] T. Kitahara, H. Nakajima and K. Okamura, "Influence of Hydrophilic and Hydrophobic Triple MPL Coated GDL on the Oxygen Transport Resistance in a PEFC under High Humidity Conditions," ECS Transactions, vol. 69, pp. 1313-1322, 2015.

[10] T. Kitahara et al, "Triple microporous layer coated gas diffusion layer for performance enhancement of polymer electrolyte fuel cells under both low and high humidity conditions," J. Power Sources, vol. 248, pp. 1256-1263, 2014.

[11] T. Kitahara, H. Nakajima and K. Mori, "Hydrophilic and hydrophobic double microporous layer coated gas diffusion layer for enhancing performance of polymer electrolyte fuel cells under no-humidification at the cathode," J. Power Sources, vol. 199, pp. 29-36, 2/1, 2012.

[12] T. Kitahara, H. Nakajima and K. Mori, "Hydrophilic and Hydrophobic Double MPL Coated GDL to Enhance PEFC Performance under Low and High Humidity Conditions," ECS Transactions, vol. 41, pp. 593-601, 2011.

[13] T. Tanuma and S. Kinoshita, "Impact of Gas Diffusion Layers (GDLs) on Water Transport in PEFCs," $J$. Electrochem. Soc., vol. 159, pp. B150, 2012.

[14] T. Tanuma and S. Kinoshita, "Impact of Cathode Fabrication on Fuel Cell Performance," Journal of the Electrochemical Society, vol. 161, pp. F94-F98, January 01, 2014.

[15] M. Ahn et al, "Influence of hydrophilicity in micro-porous layer for polymer electrolyte membrane fuel cells," Electrochim. Acta, vol. 56, pp. 2450-2457, 2/1, 2011.

[16] Y. Aoyama et al, "Effect of Wettability of Micro-Porous Layer on Microscopic Water Transport Phenomena in PEFC," ECS Transactions, vol. 64, pp. 527-535, 2014.

[17] S. Chevalier et al, "Synchrotron X-ray Radiography as a Highly Precise and Accurate Method for Measuring the Spatial Distribution of Liquid Water in Operating PEM Fuel Cells," J. Electrochem. Soc., vol. 2, pp. 107-114, 2017.

[18] N. Ge et al, "Calibrating the X-ray attenuation of liquid water and correcting sample movement artefacts during in operando synchrotron X-ray radiographic imaging of polymer electrolyte membrane fuel cells," Journal of Synchrotron Radiation, vol. 23, 2016.

[19] J. Lee, J. Hinebaugh and A. Bazylak, "Synchrotron X-ray radiographic investigations of liquid water transport behavior in a PEMFC with MPL-coated GDLs," J. Power Sources, vol. 227, pp. 123-130, 2013.

[20] R. Mukundan et al, "Effect of hydrophilic treatment of microporous layer on fuel cell performance," ECS Transactions, vol. 33, pp. 1109-1114, 2010.

[21] P. G. Stampino et al, "Effect of different substrates, inks composition and rheology on coating deposition of microporous layer (MPL) for PEM-FCs," Catalysis Today, vol. 147, pp. S30-S35, 2009.

[22] T. W. Wysokinski et al, "Beamlines of the biomedical imaging and therapy facility at the Canadian light sourcePart 1," Nuclear Instruments and Methods in Physics Research Section A: Accelerators, Spectrometers, Detectors and Associated Equipment, vol. 582, pp. 73-76, 2007.

[23] J. Hinebaugh, P. R. Challa and A. Bazylak, "Accounting for low frequency synchrotron x-ray beam position fluctuations for dynamic visualizations," J. Synchrotron Rad., vol. 19, pp. 994, 2012.

[24] S. Goswami, S. Klaus and J. Benziger, "Wetting and absorption of water drops on Nafion films," Langmuir, vol. 24, pp. 8627-8633, 2008. 